
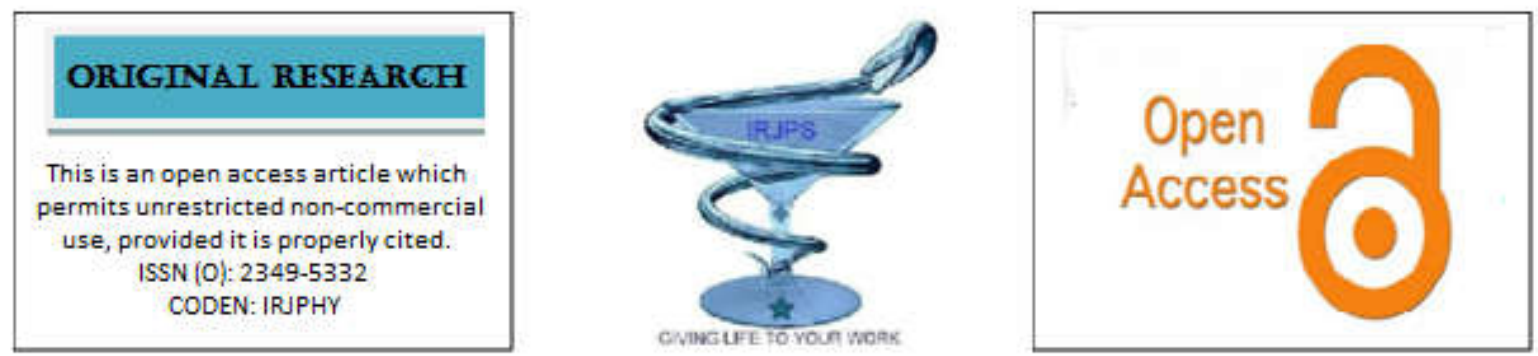

\title{
STUDY ON NUTRITIONAL AND ELECTROLYTE DISTURBANCES AND ITS MANAGEMENT IN METABOLIC DISORDER
}

\author{
L.Magimai Upgara Valan*, S. Anusha, Jithin Raj.J, M. Muthu Kumar, V. Leena Deborah \\ Department of Pharmacy Practice, C.L. Baid Metha College of Pharmacy, Chennai-60097
}

Submitted on: 20.08.19; $\quad$ Revised on: 02.09.19;

Accepted on: 05.09.19

\begin{abstract}
Metabolic disorders are the major cause of morbidity and mortality in the developing countries. The prevalence ofmetabolic disorder is common among Indian population due to their genetic and lifestyle changes. As the management involves multi drug therapy for treating metabolic disorder and its complications, both the therapy as well as the disease condition leads to various electrolyte and nutritional abnormalities in them. In this study,we observed the electrolyte and nutritional abnormalities and its management in metabolic disorder patients. The objective of the study was to categorize the metabolic disorder, assess the prevalence of electrolyte abnormalities, andits management, and rational use of nutritional supplements.A hospital based prospective observational study was conducted.123 patientsmeet the inclusion criteria in a multispecialty hospital over a period of 6months from January to June 2018. Demographic and clinical parameters were collected using a specially designed form. The data was tabulated and analysed using Microsoft excel, and graphpad prism for statistical analysis.Total number of patients was 123 , female were 71 , and male were 52. Majority of population were in the age group between 78-87 years with $27 \%$. Among metabolic disorder, endocrine and cardiovascular disorder was more prevalent with $41 \%$. The most common electrolyte abnormality was hyponatremia with $48 \%$, followed by hypochloremia with $17 \%$, hyperkalemia with $9 \%$ and others was found to be least common. Most of the abnormalities were treated with IV management with $57 \%$ followed by oral with $43 \%$. Nutritional abnormalities were studied based on the use of supplements prescribed to the patient. The most common supplement prescribed was vitamin with $36 \%$. 293 drug induced nutritional depletions were identified and among them, drugs used for treating cardiovascular and endocrine disorder caused nutritional depletion.From this prospective observational study, it was concluded that electrolyte and nutritional abnormality is common among hospitalized metabolic disorder patients. Therefore, clinical pharmacists play a key role in identifying metabolic disorder patients with these abnormalities, suggest other healthcare professionals regarding their health condition, and prescribe supplements based on these levels to reduce the burden of cost and disease condition, and improve their quality of life.
\end{abstract}

Keywords: Metabolic disorder; Electrolyte abnormalities; Nutritional supplement; Management.

Corresponding author: L. M. U. Valan

E-mail: magimaiuvalan@gmail.com

Mobile: 9003225499

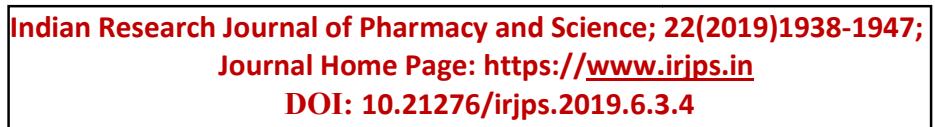




\section{INTRODUCTION}

A metabolic disorder (MD) can happen when abnormal chemical reactions in the body alter the normal metabolic process ${ }^{1}$. It can take many forms: a) Deficiency of enzyme or vitamins that is important for chemical reaction b) Abnormal chemical reaction that hinders metabolic process c) Disease in liver, pancreas, endocrine glands, and other involved in metabolism d) Nutritional deficiencies ${ }^{2}$. There are different groups of disorders. Some affect the breakdown of amino acids, carbohydrates, or lipids. Another group, mitochondrial diseases, affects the parts of the cells that produce the energy.

Fluid and electrolyte balance is a key concept to understand for maintaining homeostasis, and for a successful treatment of many metabolic disorders. There are various regulating mechanisms for the equilibrium of electrolytes in organisms. Disorders of these mechanisms that are involved in metabolic process result in electrolyte imbalances that may be life-threatening clinical conditions. Most of important electrolyte imbalances are hypo- and hyper- states of sodium, potassium, calcium, phosphorous, magnesium, and chloride. ${ }^{3}$

Hyponatremia is defined as the serum sodium concentration less than $135 \mathrm{mEq} / \mathrm{L}$ is the most common electrolyte abnormality encountered in the clinical practice. Different types of hyponatremia, Pseudohyponatremia (hyperlipidemia, hyperproteinaemia, hyperglycemia), hypervolaemic hyponatremia (body fluid loss, renal causes ), hypovolaemic hyponatremia(heart failure, chronic heart failure ), euvolaemic hyponatremia(drugs like diuretics, barbiturates, chlorpropamide, carbamazepine, hypothyroidism ) etc. Hypernatremia is defined as serum sodium concentration above $145 \mathrm{mEq} / \mathrm{L}$ and it is uncommon in normal patients and in adults exclusive due to total body water defecit. The causes include chronic renal insufficiency, hypercalcemia, hypokalemia, lithium toxicity etc. ${ }^{4}$

Hypokalemia, defined as a plasma $\mathrm{K}+$ concentration $<3.5 \mathrm{mmol} / \mathrm{L}$, may result fromone or more of the followings: decreased net intake like starvation; shift into cells like metabolic alkalosis, insulin, $\alpha 2$ Adrenergic agonist, total parenteral nutrition; and increased net loss like diarrhea, sweating, renal loss: diuretics, primary and secondary hyperaldosteronism. Hyperkalemia is defined as a plasma $\mathrm{K}+$ concentration $>5.3 \mathrm{mmol} / \mathrm{L}$, occurs as a result of either $\mathrm{K}+$ release from cells or decreased renal loss. Iatrogenic hyperkalemia may result from overzealous parenteral $\mathrm{K}+$ replacement or in patients with renal insufficiency. Metabolic acidosis, with the exception of those due to the accumulation of organic anions, can be associated with mild hyperkalemia resulting from intracellular buffering of $\mathrm{H}+$. Insulin deficiency andhypertonicity (e.g., hyperglycemia) promote $\mathrm{K}+$ shift from the ICF to the ECF. Severe digitalis toxicity and treatment with beta-blockers may contribute to the elevation in plasma $\mathrm{K}+$ concentration. Other drugs like ACE inhibitors, $\mathrm{ARBs}$ and Spironolactone are often responsible for hyperkalaemia. ${ }^{4}$

Hypermagnesemia is defined as serum magnesium concentration above $2.2 \mathrm{mg} / \mathrm{dl}$.Mostly seen in patients with renal insufficiency. The causes include can be seen with bowel obstruction, colitis, gastric dilation and use of medications that decrease motility, narcotics and anticholinergics, hypoparathyroidism, hypothyroidism. Hypomagnesemia is defined as serum magnesium concentration below $1.5 \mathrm{mg} / \mathrm{dl}$. The causes include patients maintained with diuretics, malnourished and alcoholic patients, patients with hypokalemia, patient with acute coronary artery disease and ventricular arrhythmias, patients with specific medications (aminoglycosides, amphotericin B, cisplatin). ${ }^{4}$

Hypophosphatemia is defined as mild $2-2.5 \mathrm{mg} / \mathrm{dl}$, moderate $1-2 \mathrm{mg} / \mathrm{dl}$, severe less than $1 \mathrm{mg} / \mathrm{dl}$. The causes include Vomiting, malabsorption syndromes, vitamin D deficiency, sepsis, diabetic ketoacidosis, hyperparathyroidism, diuretics, steroids, phosphate binders, xanthine derivatives, beta 2 agonists, trauma, acute renal failure etc. Hyperphosphatemia is a serum phosphate concentration $>4.5 \mathrm{mg} / \mathrm{dL} \quad(>1.46$ $\mathrm{mmol} / \mathrm{L}$ ). Causes include chronic kidney disease, hypoparathyroidism, and metabolic or respiratory acidosis. $^{4}$

Hypochloremia is defined as plasma cl $<96$ $\mathrm{mEq} / \mathrm{L}$.Loss of body fluids from prolonged vomiting, 
diarrhea, sweating or high fevers. Drugs such as: bicarbonate, corticosteroids, diuretics, and laxatives are responsible for low chloride. ${ }^{5}$ Hyperchloremia is defined as plasma cl- more than $110 \mathrm{mEq} / \mathrm{L}$.Loss of body fluids from prolonged vomiting, diarrhea, sweating or high fever (dehydration). High levels of blood sodium. Kidney failure, or kidney disorders, Diabetes insipidus or diabetic coma, Drugs such as androgens, corticosteroids, estrogens, and certain diuretics are responsible for high chloride levels. ${ }^{6}$

Hypercalcemia is usually defined as the serum calcium level above $10.5 \mathrm{mg} / \mathrm{dl}$. The causes include Endocrine (hyperparathyroidism, hyperthyroidism, adrenal insufficiency). Malignant disease (ectopic secretions of parathyroid hormone, multiple myeloma, cancer metastatic to bone), granulomatous disease (tuberculosis, sacoidosis), pharmacologic agents (vitamin $\mathrm{A}$ and $\mathrm{D}$, thiazide diuretics) Miscellaneous (dehydration, rhabdomyolysis). Hypocalcemia is a total serum calcium concentration $<8.8 \mathrm{mg} / \mathrm{dL}(<2.20 \mathrm{mmol} / \mathrm{L})$ in the presence of normal plasma protein concentrations or a serum ionized calcium concentration $<4.7 \mathrm{mg} / \mathrm{dL}$ $(<1.17 \quad \mathrm{mmol} / \mathrm{L})$. Causes include hypoparathyroidism, vitamin D deficiency, and renal disease. ${ }^{4}$

Nutritional disorders may be caused by a deficiency or excess of one or more nutrients, or by the presence of a toxin in the diet, disease, and drug therapy.They may include deficiencies or excesses in the diet, obesity and eating disorders, and chronic diseases such as cardiovascular disease, hypertension, cancer, and diabetes mellitus. Nutritional diseases also include developmental abnormalities that can be prevented by diet, metabolic disorders that respond to dietary treatment, the interaction of foods and nutrients with drugs, food allergies and intolerances, and potential hazards in the food supply. ${ }^{3}$

As metabolic disorder affects the body's ability to disrupt certain vitamins and minerals, that are essential for normal functioning of metabolic process in humans. Hence, the present study was aimed to observe the electrolyte and nutritional abnormalities in metabolic disorder patients.

\section{MATERIALS AND METHODS}

A prospective and observational study was conducted in a tertiary care hospital over a period of 6 months from January 2018 to June 2018 after getting approval from hospital ethical committe. The study includes 123 patients with metabolic disorder. The patients below 18 years, pregnant, lactating, and inherited disorders were excluded from the study. Patients with metabolic disorders such as diabetes, hypertension, thyroid disorders, parathyroid disorders, lipid disorders, obesity, and organ failure were included in the study.

Data's were collected from the patient medical record and laboratory results using a specially designed data collection form. All the data's were compiled and subjected to appropriate statistical analyses.

\section{RESULTS}

In the 123 patients with metabolic disorder, 57.2\% were female and $42.8 \%$ were male(Table1). The mean age of the patient was $57.5 \pm 24.49$ years (Table 2 ). The prevalence of both smoker and alcoholic was $22 \%$, only alcoholic was $4 \%$, smoker was $8 \%$ and no smoker and alcoholic was $67 \%$. BMI was calculated based on obesity foundation Indian category, eight (7\%) patients were found to be underweight, 33 (27\%) patients were found to be normal body weight, $47(38 \%)$ patients were found to be overweight, and 35 (28\%) patients were found to be obese.

Patients were categorized into different metabolic disorder classification. $24(20 \%)$ patients were diagnosed with endocrine metabolic disorder, 26 (21\%) cardiovascular disorder, 5 (4\%) mineral metabolic disorder, 6 (5\%) cardiovascular and mineral metabolic disorder, $51(41 \%)$ endocrine and cardiovascular disorder, $2(2 \%)$ endocrine and mineral metabolic disorder, $9(7 \%)$ had combination of endocrine, cardiovascular and mineral metabolic disorder. 
Table 1: GENDER CATEGORIZATION $(\mathrm{n}=123)$

\begin{tabular}{|ccc|}
\hline GENDER & NO OF PATIENTS & PERCENTAGE\% \\
\hline MALE & 52 & 42.8 \\
\hline FEMALE & 71 & 57.2 \\
\hline
\end{tabular}

Table 2 AGE GROUP CATEGORISATION $(\mathrm{n}=123)$

\begin{tabular}{|c|c|c|}
\hline AGE GROUP (YEARS) & $\begin{array}{c}\text { NO OF PATIENTS } \\
(\mathbf{n})\end{array}$ & PERCENTAGE \% \\
\hline $\mathbf{1 8 - 2 7}$ & 2 & 2 \\
\hline $\mathbf{2 8 - 3 7}$ & 3 & 2 \\
\hline $\mathbf{3 8 - 4 7}$ & 10 & 9 \\
\hline $\mathbf{4 8 - 5 7}$ & 11 & 21 \\
\hline $\mathbf{5 8 - 6 7}$ & 26 & 25 \\
\hline $\mathbf{6 8 - 7 7}$ & 31 & 27 \\
\hline $\mathbf{7 8 - 8 7}$ & 33 & 6 \\
\hline $\mathbf{8 8 - 9 7}$ & 7 & \\
\hline MEAN \pm SD & & $57.5 \pm 24.49$ \\
\hline
\end{tabular}

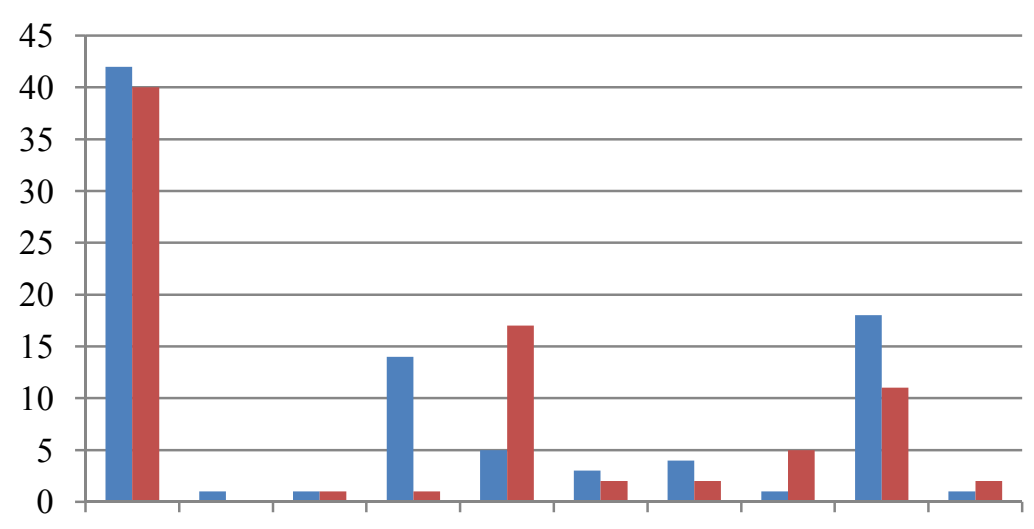

- SYMPTOMATIC

- ASYMPTOMATIC

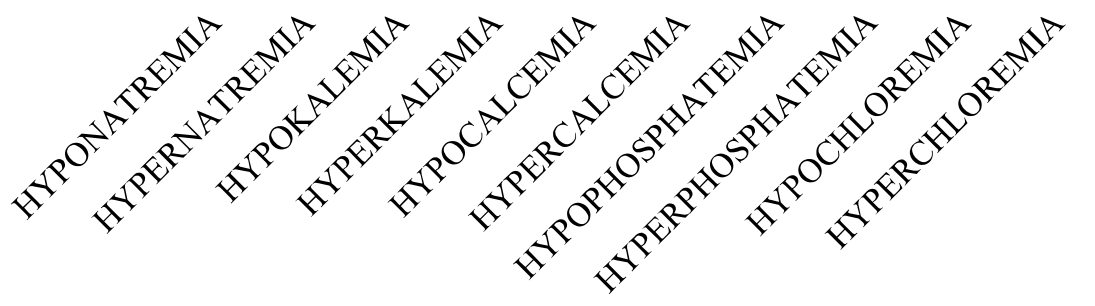

FIGURE 1: SYMPTOMATIC AND ASYMPTOMATIC DISTRIBUTION (n=171) 
Total of 171 electrolyte abnormalities were identified. Frequency distribution of electrolyte abnormalities was found to be as follows: hyponatremia $48 \%$, hypernatremia $1 \%$, hypokalemia $1 \%$, hyperkalemia $9 \%$, hypocalcemia $13 \%$, hypercalcemia $3 \%$, hypo- and hyperphosphatemia each 3\%, hypochloremia $17 \%$ and hyperchloremia $2 \%$ (Table 3 ). General characteristics like whether the patient was symptomatic and asymptomatic was identified. Out of 171 abnormalities, 90 presented with symptoms, and 81 presented without symptoms (Figure 1). Drugs used for treating electrolyte abnormality was $14 \%(n=133)$. Out of 133 drugs used, $57 \%$ was IV electrolyte and $43 \%$ was oral electrolyte. The most commonly used IV, oral electrolyte was IVF Normal saline (29\%), and tolvaptan (46\%) respectively (Table 4 and 5). 46\% patients were managed at hospital and discharged with normal electrolyte values, while $54 \%$ were treated for disease condition that corrects the electrolyte imbalance once the disease state is corrected and discharged with advice.

Table 3: ELECTROLYTE DISORDER CATEGORIZATION (n=171)

\begin{tabular}{|c|c|c|c|}
\hline \multicolumn{2}{|c|}{ CONDITION } & $\begin{array}{c}\text { NO OF CASES } \\
\text { (n) }\end{array}$ & PERCENTAGE\% \\
\hline \multirow{2}{*}{ SODIUM } & HYPONATREMIA & 82 & 48 \\
\cline { 2 - 4 } & HYPERNATREMIA & 1 & 1 \\
\hline \multirow{2}{*}{ POTASSIUM } & HYPOKALEMIA & 2 & 9 \\
\cline { 2 - 4 } & HYPERKALEMIA & 15 & 13 \\
\hline \multirow{2}{*}{ CALCIUM } & HYPOCALCEMIA & 22 & 3 \\
\cline { 2 - 4 } & HYPERCALCEMIA & 5 & 3 \\
\hline \multirow{2}{*}{ CHLORIDE } & HYPOPHOSPHATEMIA & 6 & 3 \\
\cline { 2 - 4 } & HYPERPHOSPHATEMIA & 6 & 2 \\
\hline
\end{tabular}

Table 4: IV ELECTROLYTES USED $(n=76)$

\begin{tabular}{|ccc|}
\hline ELECTROLYTES & NO OF & PERCENTAGE \% \\
\hline P\% NORMAL SALINE & 7 & 9 \\
\hline IVF.NS & 22 & 29 \\
\hline IVF.DNS & 5 & 7 \\
\hline IVF.NS/RL & 14 & 18 \\
\hline IVF.PLASMALYTE & 11 & 14 \\
\hline INJ.KCL & 9 & 12 \\
\hline INJ.ISOTONIC BICARBONATE & 2 & 3 \\
\hline INJ.MGSO4 & 2 & 3 \\
\hline INJ.NAHCO3 & 1 & 1 \\
\hline INJ.CALCIUM GLUCONATE & 3 & 4 \\
\hline
\end{tabular}


Table 5: ORAL ELECTROLYTES USED $(n=57)$

\begin{tabular}{|ccc|}
\hline ELECTROLYTES & $\begin{array}{c}\text { NO OF PRESCRIPTIONS } \\
(\mathbf{n})\end{array}$ & PERCENTAGE\% \\
\hline SYP.KCL & 19 & 33 \\
\hline K BIND SACHET & 5 & 9 \\
\hline TOLVAPTAN & 26 & 46 \\
\hline SODIUM BICARBONATE & 4 & 7 \\
\hline SEVELAMER & 2 & 3 \\
\hline CALCIUM ACETATE & 1 & 2 \\
\hline
\end{tabular}

Nutritional abnormalities was analysed based on the supplement prescribed to the patient. $19 \%(\mathrm{n}=187)$ of supplements were used for treating nutritional abnormalities. Nutritional supplements were classified as vitamins and minerals (Table 6). $36 \%$ of patients received vitamins, $9 \%$ minerals, $35 \%$ combination of vitamins and minerals, and $20 \%$ no supplements. Out of 187 nutritional supplements, oral and IV supplement usage was $89 \%$ and $11 \%$ respectively (Table 7). 293 drug induced nutritional depletion was identified (Table 8). Out of which 160 drug - nutrition interaction was identified in drug prescribed for treating endocrine and cardiovascular disorder. Nutritional depletion management was analysed based on prophylactic and therapeutic use of supplements. $41 \%$ were used as prophylactic, $15 \%$ as therapeutic, $22 \%$ for both prophylactic and therapeutic and $22 \%$ did not receive any supplements (Table 9).

Table 1: NO OF PRESCRIPTION CONTAINING NUTRITIONAL SUPPLEMENTS (n=123)

\begin{tabular}{|c|c|c|}
\hline SUPPLEMENTS & $\begin{array}{c}\text { NO OF PRESCRIPTION } \\
\text { (n) }\end{array}$ & PERCENTAGE \% \\
\hline VITAMINS & 44 & 36 \\
\hline MINERALS & 11 & 9 \\
\hline $\begin{array}{c}\text { VITAMINS + } \\
\text { MINERALS }\end{array}$ & 43 & 35 \\
\hline NONE & 25 & 20 \\
\hline
\end{tabular}

Table 7: NUTRITIONAL SUPPLEMENT USAGE $(n=187)$

\begin{tabular}{|c|c|c|}
\hline MANAGEMENT & $\begin{array}{c}\text { TOTAL } \\
\text { (n) }\end{array}$ & $\begin{array}{c}\text { PERCENTAGE } \\
\text { \% }\end{array}$ \\
\hline ORAL MANAGEMENT & 167 & 89 \\
\hline IV MANAGEMENT & 20 & 11 \\
\hline TOTAL & 187 & 100 \\
\hline
\end{tabular}


Table 8: DRUG- NUTRITION INTERACTIONS (n=293)

\begin{tabular}{|c|c|}
\hline CONDITION & DRUG- NUTRITION INTERACTIONS \\
\hline Endocrine Disorder & 31 \\
\hline Cardiovascular Disorder & 65 \\
\hline Endocrine + Cardiovascular Disorder & 160 \\
\hline Mineral + Cardiovascular Disorder & 9 \\
\hline Endocrine, Cardiovascular \& Mineral Metabolic \\
Disorder
\end{tabular}

Table 2: MANAGEMENT OF NUTRITIONAL DEPLETION (n=123)

\begin{tabular}{|c|c|c|c|c|c|}
\hline CATEGORY & PROPHYLACTIC & THERAPEUTIC & $\begin{array}{l}\text { PROPHYLACTIC } \\
\text { + THERAPEUTIC }\end{array}$ & NONE & TOTAL \\
\hline ENDOCRINE & 15 & 2 & 2 & 5 & 24 \\
\hline CVS & 7 & 3 & 10 & 6 & 26 \\
\hline MINERAL & 1 & 3 & - & 1 & 5 \\
\hline CVS + MINERAL & 1 & 1 & 2 & 2 & 6 \\
\hline ENDOCRINE + CVS & 23 & 7 & 11 & 10 & 51 \\
\hline $\begin{array}{c}\text { ENDOCRINE + } \\
\text { MINERAL }\end{array}$ & 1 & 1 & - & - & 2 \\
\hline $\begin{array}{c}\text { ENDOCRINE + CVS } \\
\text { +MINERALS }\end{array}$ & 2 & 2 & 27 & 3 & 9 \\
\hline $\begin{array}{c}\text { TOTAL } \\
\text { PERCENTAGE } \\
\%\end{array}$ & 50 & 19 & 22 & 22 & 100 \\
\hline
\end{tabular}

\section{DISCUSSION}

Theprincipal results indicates that electrolyte and nutritional abnormalities are more prevalent common in metabolic disorder patients ranging from age group between 78-87 years followed by 68-77 years of age and the prevalence of metabolic disorder are low in age group of 18-27 years of age. In this study, when compared to male $(42.8 \%)$, females $(57.2 \%)$ are slightly having higher chance of developing metabolic disorder. Statistics conducted by Women's Health USA 2009. Rockville, Maryland: U.S. Department of Health and Human Services, 2009 was also same [2]. As per this study, male with social history of both smoking and alcoholic were high which increases the risk of metabolic disorder in males. While $23 \%$ were not related with conception of smoking and alcohol. However, female patients have reported with highest percentage of metabolic disorder in this study, which may not be relatable to social factors and may be relatable to other factors like hormonal imbalances as they are more prone and sensitive to hormonal changes. The BMI was classified based on obesity foundation Indian category. Less than 18.5 were classified as underweight, range between 18.50- 24.9 were classified as normal body weight, greater than 25 were classified as over-weight and, greater than 30 were classified as obese. From the present study, we observed that overweight patients were more prone to have metabolic disorder followed by obese patients. Study conducted by Sharleen Sidhu also observed the same et al., ${ }^{7}$

Endocrine disorders such as diabetes mellitus, hypothyroidism, and cardiovascular disorders such as hypertension were more prevalent in this study when compared to other metabolic disorder. As Diabetes 
and hypertension, share common pathways and these pathways interact and influence each other. Hypertension and diabetes are both results of the metabolic disorder. They may, therefore develop one after the other in the same individual. The same was observed by Guido Lastra and Bernard M. Y. Cheung et al., ${ }^{8,9}$

Out of 984 drugs used in 123 prescriptions, 19\% were drugs for nutritional abnormalities, $14 \%$ were drugs for electrolyte abnormalities, and $67 \%$ of drugs were used for treating metabolic disorder and its complications.

In sodium disturbance, hyponatremia was found to be in higher percentage (48\%) which was previously observed in studies conducted by qazi najeeb et al.,[10]. In these, moderate hyponatremia was common among them with $54 \%$ which was previously observed in studies conducted by Corona Get al. This study concludes that moderate hyponatremia was associated with an increased risk of mortality in metabolic patients. Corona Get al., concluded the same. ${ }^{10}$

In potassium disturbance, hyperkalemia was common among them with $88 \%$ which was previously observed in studies conducted by Qazi najeeb et al., ${ }^{10}$ and hypokalemia was least among them with $12 \%$ while other studies concluded that hypokalemia was most common among than hyperkalemia. This study concludes that diabetes and hypertensive patients were more prone to develop hyperkalemia than hypokalemia. Marsha A. Raebel et al., concluded the same. ${ }^{12}$

In calcium disturbance, hypocalcemia was common among them with $81 \%$ and hypercalcemia was least among them with $19 \%$. The same was concluded by qazi najeeb et al.,[10]. Hypocalcemia might be one of the risk factors for developing cardiovascular disease such as hypertension. Mi-Hyun Kim et al., ${ }^{13}$ concluded the same.

There is no difference between the hypo- and hyperphosphatemia percentage, while other studies concluded that patients with metabolic disorder such as hypertension, diabetes were more prone to develop phosphorous disturbances which was conducted by fang L et al. ${ }^{14}$ Hypochloremia was more prevalent than hyperchloremia, which indicates that diabetes group in metabolic disorder patients are more prone to develop diabetic acidosis. Study done by Anupriya Sharma et al., alsoconcluded the same. ${ }^{15}$

In nutritional supplements, highest percentage of vitamins, followed by both vitamins and minerals were prescribed in metabolic disorder patients, which suggests that both vitamin and mineral loss occurs in metabolic disorder patients either due to disease condition or drug therapy involved. In 123 prescriptions, 293 drug interactions were identified. The drugs that were prescribed for both diabetes and hypertension cause nutritional loss more when compared to other drugs prescribed for other conditions. This suggests that nutritional loss should be kept in mind while prescribing anti-diabetic and anti-hypertensive drugs. Routine monitoring of vitamin $b_{12}$ and complete blood count should be done in both diabetes and hypertension patients Jessica Barbieri et al., Adnan Khan et al., concluded the same. ${ }^{16,17}$

\section{CONCLUSION}

The present study concludes that patients more than 60 years irrespective of gender were more prone to develop metabolic disorder. Endocrine and cardiovascular metabolic disorder was predominant. Among metabolic disorder complications, nutritional and electrolyte abnormality is the common cause for hospitalization.

Hyponatremia was more common among them and was managed with both IV and oral electrolytes. These disturbances should be monitored, diagnosed, and managed correctly during hospitalization to avoid increased length of hospitalization and reduce morbidity and mortality of patients. Hence, monitoring of these electrolytes during routine screening of metabolic disorder patients will be of great benefit in subjects suffering from metabolic disorder. In metabolic disorder specifically diabetes, hypertension and hypothyroid patients were more vulnerable to develop nutritional loss like calcium, vitamin $\mathrm{B}_{12}$, folic acid, $\mathrm{CoQ} 10$, iron either due to the drug therapy involved in disease condition or the 
disease state itself.

Therefore, clinical pharmacists should identify metabolic disorder patients with these abnormalities and suggest other healthcare professionals regarding patient health condition and prescribing supplements based on these levels to reduce the burden of cost and disease condition, and improve their quality of life.

\section{ACKNOWLEDGEMENT:}

This is a self-funded study. The authors are thankful for the support by Head of the Department of Pharmacy Practice and Principal of C. L. Baid Metha College of pharmacy.

\section{REFERENCES:}

1. Medline plus. [Internet].vs. National institute of health; 23 august 2016. [31.1.2018] Available from. URL: https:// medlineplus.gov/metabolic .html.

2. HRSA [internet]. Women health USA; 2009(2/9/18). Available from URL: htpps://mchb.hvsa.gov/whvsaoa/hstat/hl/pa ges $/ 217$ emd.html.

3. Weininger J. Nutritional disease [Internet]. Encyclopædia Britannica. Encyclopædia Britannica, inc.; 2018 [cited 2018Dec31]. Available from: https://www.britannica.com/science/nutritio nal-disease

4. Camiron L.P tennig and Corey meslovis. Medicine and surgery: metabolism and endocrinology $\quad .8^{\text {th }}$ edition .uk. Elsevier; 2013.

5. Cleveland Clinic Cancer Hypochlorecmia (Low Chloride) [Internet]. DexamethasoneDrug Information - Chemocare. [Cited 2018Dec31]. Available from http://chemocare.com/chemotherapy/sideeffects/hypochloremia-low-chloride.aspx

6. Cleveland Clinic Cancer Hyperchlorecmia (High Chloride) [Internet]. DexamethasoneDrug Information - Chemocare. [Cited 2018Dec31]. Available from http://chemocare.com/chemotherapy/sideeffects/hyperchloremia-low-chloride.aspx
7. Sidhu s.parikh T, Burman KD. Endocrine changes in obesity [internet], pub med; available

from:URL.https://www.ncbi/:nlmonih.gov/b ooks/NBK27905.

8. Guido Lastra, Sofia Syed, Romayne Kurukulasuriya, Camilla Manrique James, R. Sowers. Type 2 diabetes mellitus and hypertension: An update. Type 2 diabetes mellitus and hypertension: An update. Endocrinol Metab Clin North Am. 2014; 43(1): 103-122.

9. Bernard M. Y. Cheung\&Chao Li. Diabetes and Hypertension: Is There a Common Metabolic Pathway? Curr Atheroscler Rep 2012; 14:160-166.

10. Jaskiran kaur, naved Ahmed, Akash gupta. Changes in the electrolyte profile of patients having hypothyroidism med sci cli res, 2015; 13:28-32.

11. Giovanni Corona, Corinna Giulian. Moderate Hyponatremia Is Associated with Increased Risk of Mortality: Evidence from a Meta-Analysis. PLoS ONE 8(12): e80451

12. Marsha A. Raebel, PharmD, Christopher M. Blanchette, PhD. Diabetes and DrugAssociated Hyperkalemia: Effect of Potassium Monitoring. JGIM, 2010; 2:2628.

13. Mi-Hyun kim, Mi-lyeong choi. Daily calcium intake and its relation to blood pressure ,blood lipids and oxidative biomarkers in hypertensive and normotensive subjects ,nutr respract,2012;6(5):421-428

14. Fang L. Level of serum phosphorous and adult type 2 diabetes mellitus.2016; 41(5):502-6.

15. Anupriya $S$,Hirulkar N.B., Ranka P. Effect of hyperglycemia on electrolyte imbalance .Int J pharm bio ,2011;2(1):526-533.

16. Barbieri J. Paula c.fontela, matias N.frizzo. Anaemia in patients with type 2 diabetes mellitus. Hind pub cov 2015; Article ID 354737.

17. Adnan khan ,batik I, Mohammed H.shah 
.prevalence of vitamin B12 deficiency in patients with Type 1 diabetes mellitus on metformin : A study from Khyber

pakhtunthwa. Inl J Med ,2013;1-5 